\title{
Special issue: When social media meets physical world
}

\author{
Rongrong $\mathrm{Ji}^{1} \cdot$ Yue $\mathrm{Gao}^{2} \cdot \mathrm{Qi} \mathrm{Tian}^{3} \cdot \mathrm{Qionghai}^{\mathrm{Dai}}{ }^{4} \cdot$ Ralf Steinmetz $^{5}$
}

Published online: 8 December 2015

(C) Springer-Verlag Berlin Heidelberg 2015

With the advent of Web 2.0 era, there is an explosive growth of social media data available on user-sharing websites such as Flickr, Youtube, and Zooomr. Besides the plain visual signals, more and more social media data are now associated with rich location context such as GPS tags, location identifications, or geographical and cultural cues, which benefit a wide variety of potential location-based multimedia applications such as landmark identification, location recognition, tourism recommendation, as well as photographing suggestion. Such unique combination of social and geographical tagging enables a more seamless perspective to dig out valuable insights from the user-sharing multimedia corpus in a large scale. Especially, coming with the ever growing popularization of smartphone cameras as well as instant message sharing services such as Twitter and Foursquare, there is a great potential for exploring cheaply available, large-scale social multimedia to assist and facilitate location related computing and services. Recent years have witness a great popularity of researches on the location-based multimedia modeling, learning, and application in social media analysis. Such location information makes the traditional difficult problems in multimedia content analysis become more tractable. For example, large-scale image repository can be significantly pruned if we use the

Rongrong $\mathrm{J}$

jirongrong@gmail.com

Xiamen University, Xiamen, China

2 National University of Singapore, Singapore, Singapore

3 University of Texas at San Antonio, San Antonio, USA

4 Tsinghua University, Beijing, China

5 Technische University Darmstadt, Darmstadt, Germany
GPS information to filter out the irrelevant images with respect to the query image.

Recently, more and more research efforts have been dedicated to the aforementioned challenges and opportunities. Especially in the past three years, related papers are extensively published in ACM MM, SIGIR, WWW, and CVPR. Yet, a special issue focusing on this specific topic on related journals is still missing so far. Therefore, we see a timely opportunity to organize a special issue to bring together active researchers to share recent progress in this exciting area. Our goals are three-fold: (1) theories and applications on social multimedia computing related to location services; (2) survey on the progress of this area in the past years; (3) discuss emerging applications based on this newly scenarios.

With a very selective review process, eventually in total 14 papers are selected into our special issue. One core problem in social media computing is the problem of image tagging. To handle the problem of image tagging in social media data, Nie et al. (Geo-location driven image tagging via cross-domain learning) introduced a geo-location driven image tagging scheme via cross-domain learning. The work by Li et al. (On the tag localization of web video) further extends the problem of geo-aware social media tagging from image domain to the video domain. Another work from Sun et al. (Social video annotation by combining features with a tri-adaptation approach) further proposed a novel social video annotation approach that combines multiple feature sets based on a tri-adaptation approach. In addition, the work by Liu et al. (Detecting and tagging users' social circles in social media) presents a general algorithm for detecting users real friends in social media and dividing them into different social circles automatically according to the closeness of their relationships. Besides the linking across modalities (tagging and 
visual content), the linking among data is another key issue in this field. In (Linking socially contributed media with events), Liu et al. presents a method combining semantic inferencing and visual analysis for automatically finding media (photos and videos) illustrating events. Their scheme reports on experiments validating our heuristic for mining media sharing platforms and large event directories in order to mutually enrich the descriptions of the content they host.

At the social media side, food has become one of the highest rated topics with the popularity of mobile devices. Texts, photos, and videos about various cuisines are produced when people tweet, blog, and interact with friends in the social networks in their daily life. On the other hand, tourists often encounter cuisine names that they have difficulties in understanding the meanings, even with the help of a dictionary. Ming et al. (Resolving local cuisines for tourists with multi-source social media contents) present a novel scheme to resolve the local cuisines for tourists with multi-source social media contents. Geo-related social media data are further introduced for the task of location landmark recognition. In the work of $\mathrm{Li}$ et al. (Mining location-aware discriminative blocklets for recognizing landmark architectures), a location-aware building recognition model is introduced using locklets, i.e., spatial adjacent blocks associated with their relative positions. In addition, the work from Min et al. (An incremental probabilistic model for temporal theme analysis of landmarks) further propose a novel Incremental Spatio-Temporal Theme Model (ISTTM), which can incrementally mine temporal themes that characterize the temporal information of landmarks.

Sentiment assessment is another raising topic in the computing of geo-related social media. In the work by Cao et al. (A cross-media public sentiment analysis system for microblog), a cross-media public sentiment analysis system is introduce to assess the sentiments for microblog. Another hot topic is the so-called question answering in social media data. In (Question microblog identification and answer recommendation), Liu et al. further presented a novel scheme to mine accurate answers from questionsanswering corpus.

Visual search retains as one of the most important topics in social media analytics. In this special issue, we first introduce the work from Xia et al. (Geometric discriminative features for aerial image retrieval in social media) that adopts geometric discriminative features for aerial image retrieval in social media. Then, we introduce the work from Cheng et al. (The effects of multiple query evidences on social image retrieval) to analyze the effects of multiple query evidences on social image retrieval. Both papers give systematic analysis on how the image search problem is challenging and where is the potential solution to improve image search system in the context of social media data. In $\mathrm{Li}$ et al. (Fast verification via statistical geometric for mobile visual search) a statistical scheme is presented to replace RANSAC in scenario where fast spatial verification is needed.

Nowadays, microblogging platforms such as Twitter and Weibo can also be seen as a good media to present reviews about topics. In the last paper of this special issue Wang et al. (A location-aware TV show recommendation with localized semantic analysis) studies the application of location-aware TV show recommendation, which can predict whether a TV show will be popular at certain locations. 